\title{
ENGINEERING BIOMECHANICS - A COMPONENT OF InTERnational EduCATIONAL Project "FESTO-SYNERGY"
}

\author{
Alexander Poliakov ${ }^{\mathrm{a}}$, Yevgen Pashkova ${ }^{\mathrm{a}}$ Branko Katalinic ${ }^{\mathrm{b}}$, \\ Mykhaylo Kalinin $^{\text {a }}$, Volodymyr Pakhaliuk ${ }^{\mathrm{a}}$ \\ ${ }^{a}$ Sevastopol State University, Universitetskaya str., 33, Sevastopol, 299053, Russian Federation \\ ${ }^{b}$ Vienna University of Technology, Austria
}

\begin{abstract}
The modern concept of automation production uses the principles of integration of the element base at the level of systems that allows to create universal and specialized automated technological systems for solving various technological problems. Such integration has led to the appearance and development of a new area of science and technology - mechatronics. A characteristic feature of mechatronics is a synergistic unity of energy and information streams aimed at achieving a common goal. Within the framework of the International Project «FESTO-SYNERGY" in the Laboratory of Biomechanics of the Sevastopol State University was created a number of test devices for the modeling of movements of human skeleton elements. The use in these devices mechatronic modules FESTO has allowed to make them universal and to provide complex laws of motion of the executive bodies, the implementation of which is required for simulating of natural human movements as a biomechanical system.
\end{abstract}

Keyword: Automation; mechatronics; engineering biomechanics; biomechanical simulations; uncertainty; synergy; biomedical implant
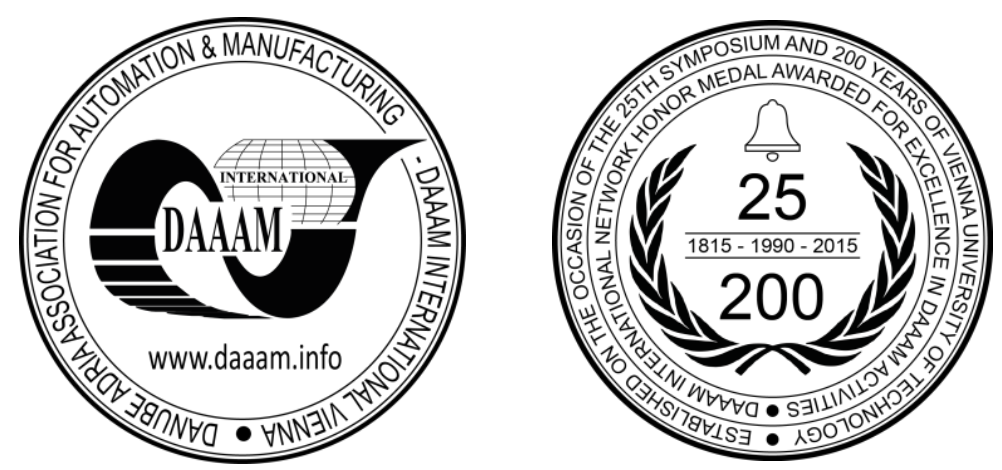

This Publication has to be referred as: Poliakov, A[lexander]; Pashkov, Y[evgeny]; Katalinic, B[ranko]; Kalinin, M[ykhaylo] \& Pakhaliuk, V[olodymyr] (2016). Engineering Biomechanics - A Component of International Educational Project ""FESTO-SYNERGY"", Proceedings of the 26th DAAAM International Symposium, pp.0350-0357, B. Katalinic (Ed.), Published by DAAAM International, ISBN 978-3-902734-07-5, ISSN 1726-9679, Vienna, Austria DOI:10.2507/26th.daaam.proceedings.047 


\section{Introduction}

The development and implementation of modern Technologies and Equipment requires the creation of innovative educational programs and projects with the use of a real industrial components and corresponding methodical support. An example are industrial-educational programs implemented within the framework of an international network project «SYNERGY», created by a number of universities together with the concern «FESTO» in October 2006, which in 2015 was transformed into a project «FESTO-SYNERGY», due to the expansion of the membership and the increasing role of the concern in enhancing the technical component of the project.

Realized by the project the concept of automation production is fully consistent with the principles of integration at the level of systems, which allow to create modern flexible automated technological complexes for solving various technological problems. As it is known, such integration has led to the appearance and development of a new area of science and technology - mechatronics, oriented to the solving problems of analysis and synthesis of mechatronic systems. A characteristic feature of such systems is a synergistic association of mechanical, electrical, electronic, information and software tools aimed at achieving a common goal.

The main objective of the project «FESTO-SYNERGY» is the intensification of practical and scientific training of qualified specialists in the field of creation and operation of mechatronic systems in various branches of industry in enterprises with a high level of automation. A special place in it allocated to the engineering biomechanics. Just at biomechanics first was used the term "synergy", which defines the process of system functioning, as a result of which the effect of the combined action of several subsystems substantially exceeds the cumulative effect of the independent actions of each individual subsystem. The famous biomechanist N. Bernstein used this term to explain the process of self-synchronization of muscles at carrying out by human, required motor actions [1]. However, unfortunately these synchronization mechanisms he could not explain. H. Hakley, which is rightly considered as founder of synergetics as a new field of science, the term "synergy" uses in the sense of "coordination", "concerted action", but also, like Bernstein, does not define the conceptual basis of this notion [2, 3]. Nevertheless, attempts to explain the essence of synergetic processes observed in the animate and inanimate nature, with the aim their possible use in complex technical systems not stopped and currently [4].

Actual tasks of engineering biomechanics are: development and study of artificial joints that provide required dynamics of human movements for a long period of time; the development of assistive devices that promote effective rehabilitation of patients with disorders functions of the musculoskeletal system; development of artificial limbs that allow people with disabilities to overcome the serious psychological traumas and to return to the active lifestyle; development of test devices for simulating biomechanical motions and others. The effectiveness of solving these problems is largely determined by the quality of necessary biomechanical information, for the obtaining, systematization and using of which in the Sevastopol State University created and operates for more than 20 years the Laboratory of Biomechanics.

One of the activities of laboratory are researches in the field of medical engineering and biomechanics in frames of project "FESTO-SYNERGY". At the development of methodologies for such studies is necessary to take into account the exceptionally complicated structure of biomechanical systems (including - human), insufficient number of reference points and the high uncertainty level, which complicates the evaluation even of external parameters of research objects. In the investigation of dynamic characteristics, in evaluation of strains, stresses and strength of individual elements of biomechanical systems methodological difficulties are increased.

In the engineering biomechanics are widely used methods of physical modeling different biological systems and subsystems, practical application of which is associated with creation of artificial organs for the orthopedic practice and their using in clinical medicine. Particular attention is paid to the study characteristics of biological organs and tissues, formulation of requirements for their artificial analogues, the implementation of which allows to provide the safety of the implantation into a living organism and to guarantee reliable operation of over a long period of time.

The aim of this work is the creation on the base of methods of system analysis and synthesis series of artificial implants and ancillary medical devices for the treatment and rehabilitation of patients with functional disorders of the locomotor apparatus, and test devices for their standard and non-standard tests. The practical realization of this aim became possible as a result of close cooperation of technological, research, medical and educational organizations in the framework of the International University Networking Project «FESTO-SYNERGY».

\section{System analysis and synthesis of artificial biomechanical systems}

\subsection{Conceptual bases of synthesis}

In solving the problems of the engineering biomechanics set forth above, should be used the system properties of biological analogues of artificial biomechanical objects. In particular, currently is known many hypotheses about the optimality of biological objects and systems formed by them. One of them amounts to the fact that in the process of natural selection continuously improved their genetic structure a relatively properties that are closely related to reproductive success. It is believed that in many cases these improvements actually has resulted to their optimal structure [5]. At this, organisms possessing biological structure, optimal in terms of natural selection, are optimal also in the sense that they minimize some estimation function, which determined by basic characteristics of the environment 
[6]. For example, N. Rashevskiy noted that "... the structure of organism is such that organism performs its functions adequately, and with a minimum consumption of energy and material required for the construction of its structures" [7].

It must be noted that the general conception of optimality biological systems is not developed up to the present time, mainly because mechanisms of global optimization during evolution is still poorly understood. In this regard, biological objects are represent as systems in the structure of which trying to find invariant characteristics, with respect to some changes, which can be considered as their own characteristics. The mathematical content of this approach is concluded, for example, in the utterance of H. Weyl, who noted that "....whenever you have to deal with some object, which endowed with the structure, try to determine its group of automorphisms, i.e. a group, whose elements are transformations, leaving without changing all structural relations. You can count on the fact that in this way you will be able to penetrate deeply into the internal structure of the object" [8]. That is, in most cases, the criteria of optimality of biological objects can be recognized as valid if they are with a high probability are not contradict with conclusions obtained by the analysis of empirical data. Thus, regularities, detected by the analysis, may interpreted as a result of the optimal inclusion of biological object into the environment.

As before, at present time, same way as is customary in physics, largely due to detailed justification and successful experience in the using, the criteria of optimality in biology and biomechanics, for the most part, based on the energetical formulation of physiological problems. I.e., is assumed the energetical optimality of living systems in relation to their functions [9]. There are many scientific works in which biologists evolutionist have used the principles of minimum cost mechanical or metabolic energy in the study of various forms of behavior in the life of animals, such as ways of movement, food extraction, competition, cooperation, reproduction $[10,11,12]$. The analysis of used mathematical models, as well as a comparison of the solutions obtained with the experimental data leads to the conclusion about their effectiveness. In this connection, accepting the human and other biological objects, as well as their some parts, as some technical devices, it can be assumed, that their structures, kinematic and dynamic parameters, etc., satisfy some, perhaps contradictory quality criteria, that may be used, for example, in the construction of anthropomorphic manipulators, prosthetic limbs, exoskeletons and similar devices, However, to date, sufficient justification of such criteria does not exist.

On the other hand, one should not consider that biomechanical devices that most closely mimic the human or any of elements of its structure are most optimal technical devices of this type, destined to implement a specific task. In some cases, these devices are really close to optimal (e.g., bioprostheses of human limbs), in others - their structure, basic parameters and control systems in one degree or another must differ from biological analogs. As noted by S. Petukhov, "...the study of symmetry in biological bodies reveals the fact that not everyone in the structure of biological organs is determined by the criterion of optimum adaptation to the implementation of direct functions, but that the important role belongs to principles of crystallization of living matter in frames of physical laws of morphogenesis [13]. In other words, as a result of the evolution biological objects are improving, getting the opportunity to function optimally, but this process is carried out within the frames of morphogenetic laws.

At the same time, it should be noted that in most studies of kinematic organization of humans and other biological systems, authors makes the emphasis on functional adaptation of objects to the environment. If this approach used, for example, when searching for optimal variants of designs of mechatronic systems, the range of possible solutions will significantly narrowed. In the previously cited monograph [13] S. Petukhov rightly notes, "... that the constructor of machines at the decision of design problems is in the best position compared to the wildlife, because he can emanate entirely from functional criterions of optimality and not be bound by necessity to comply with additional conditions arising from purely biological laws of nature".

Thus, it can argued that as a conceptual framework of synthesis of artificial biomechanical objects and systems can be used natural criteria of quality, which inherent biological counterparts. However, is necessary take into account the fact that not all of these criteria are appropriate for synthesis technical device. Firstly, some of the properties of biological objects may be superfluous for biomechanical artificial objects; secondly, in artificially created devices is not always possible to use materials similar biological tissues; thirdly, technical devices can a long period of time operate at maximum possible loads, so as in most cases is not peculiar to living systems and so on.

\subsection{Synthesis of implantable constructions}

For the treatment and rehabilitation of patients with functional disorders of the musculoskeletal system is currently used many different implantable constructions that allow fully or partially replace dysfunctional natural organs. For instance, to stabilize the spine and decompression of its structures are used interbody implants - cages or artificial intervertebral discs.

The process of implantation of stabilizing devices must considered at the system level. It connected primarily with the fact that there are various implantation techniques, based on the front (ALIF), the lateral (TLIF) and the posterior (PLIF) access to the spine. At this are used fundamentally different designs of implants. If in ALIF is possible the implantation of fully functional artificial intervertebral disks, then in PLIF the most appropriate to use implants destined primarily for decompression of spinal structures. However, the quality of surgical treatment of patients largely determined by traumatism of operation, which in the PLIF access is significantly lower. Thus, at the system level, in many cases, given the condition of the patient as the biological subsystem, PLIF is preferred compared to other methods. 
Taking into account the structural, geometric and mechanical characteristics of vertebrae for the PLIF, we proposed the design of interbody implant (cage) prismatic form, support surfaces of which provided with teeth, which prevent the migration of cage relative surfaces of vertebrae. To improve integration with bone structures the cage is sprayed hydroxyapatite ceramics (Fig.1a). Supporting surfaces of cage are rotated relative to each other at the angle of 8-12 degrees to provide the natural lordosis of the spine after implantation at the level of lumbar department (Fig.1b). In the process of rehabilitation of the patient in the postoperative period, the teeth being introduced into vertebral endplates and the pressure on them is gradually distributed over the area almost equal to the contact area of cage (Fig.1c). Roentgenograms demonstrating the stabilization of the spine by cages of this design on two levels are shown in Fig.2.

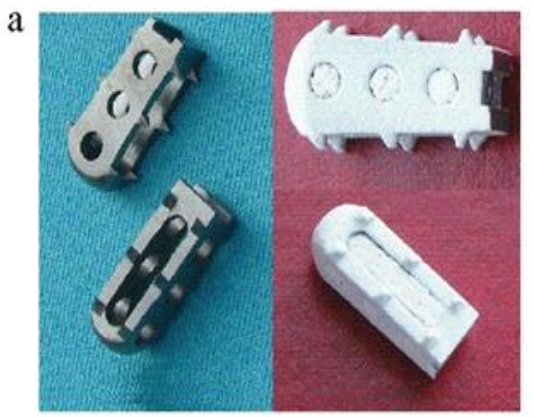

b

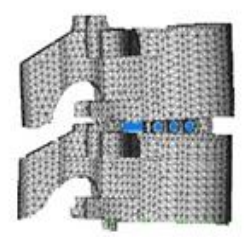

c

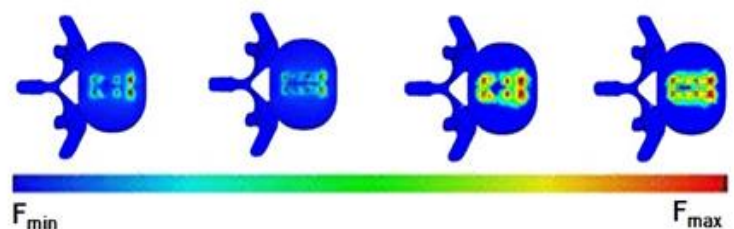

Fig. 1. (a) prismatic cage, sprayed hydroxyapatite ceramics; (b) 3D-model of the cage after replacement of the intervertebral disc; (c) modeling the process of interaction cage with vertebral endplates

Results of clinical trials suggest that the use at PLIF cages having described above parameters is justified from many points of view and contributes to reliable stabilization of damaged spine areas. However, in some cases it is impossible to use such cages. For example, at the explicit osteoporosis the cage having the relatively small supporting area can fall inside the vertebra, which leads to necessity of re-use of surgical and other treatment technologies. One way of solving this problem is the increasing supporting surfaces of cage until natural surface sizes of intervertebral disc. However, such cages almost impossible to implant by PLIF, because channel dimensions for its implantation limited by anatomic features of spine. To solve this problem we have developed multimodular cage, specialized surgical instruments and a minimally invasive technology of its implantation [14]. In Fig. 3 shows the basic steps of the implantation process (Fig. 3a) and the cage arrangement on the endplate of vertebra after implantation (Fig. 3b).
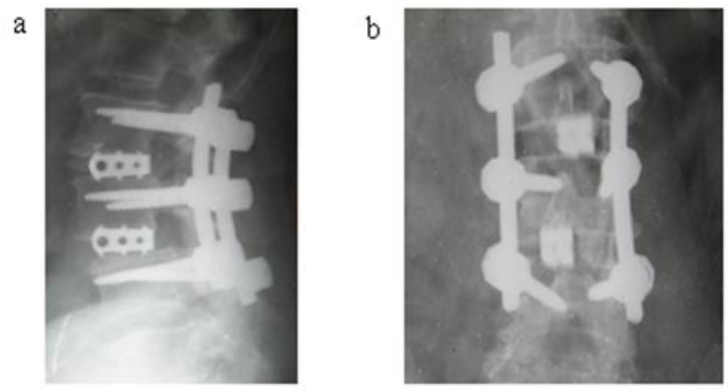

Fig. 2. X-ray patterns of patient $\mathrm{N}$ after Posterior Lumbar Interbody Fusion in two levels with prismatic cages and instrumental fixing by system "BRIDGE" (Dr. A. Bryekhov, Simferopol, Crimea, RF): (a) side view; (b) back view
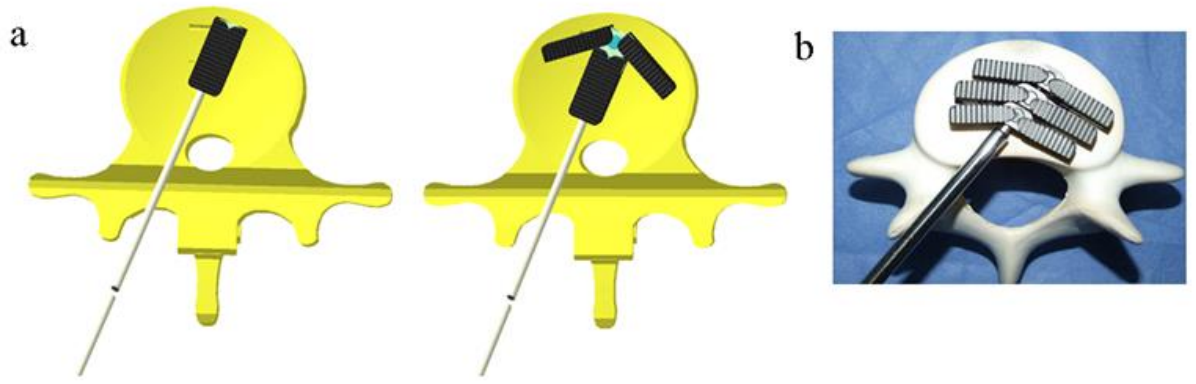

Fig. 3. (a) the sequence of basic operations at the cage implantation; (b) the cage arrangement on the endplate of vertebra after implantation 
The total area of the support surface of cage reaches more than $950 \mathrm{~mm} 2$, that allow significantly reduce stresses and unevenness of their distribution on the contact surfaces of vertebrae. One of the most significant advantages of this design is the ability to use minimally invasive techniques during its implantation. According to our estimates, obtained by computer simulation, the use of such cages in the PLIF will significantly improve the quality of patients life in the postoperative period.

The PLIF - is the standard surgical procedure, which requires the execution of a number of mandatory requirements, one of which consists of the need the localization of the damaged areas of spine with using spinal fixation systems, which commonly include longitudinal and transverse supporting rods, pedicle screws and guide elements. An analysis of the functioning of such systems shows that their elements work in conditions of complex stress-strain state, which varied in time (Fig. 4) [15].

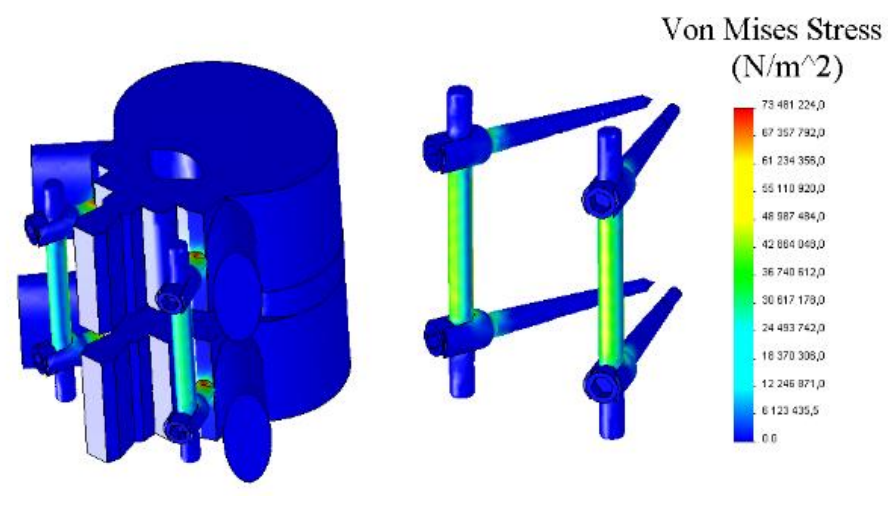

Fig. 4. The painting of stress distribution in the form of Mises on surfaces of elements of transpedicular systems

Quite often, the screws and bearing rods being destroyed by the action of dynamic loads. At this, there is a serious problem connected with removing of destroyed screws from vertebral bodies. One way of its solution is to use screws with special design elements, which required directly for their removal without destruction of bone structures [16]. We have used such approach in the system synthesis of total hip endoprosthesis [17], as well as in the synthesis of other implanted structures, which can lose strength in the process of work.

Considering the process of treatment and postoperative rehabilitation of patients with functional disorders of the musculoskeletal system at the system level, each subsystem must be considered, including, at the level of its interaction with other subsystems. In sense of synergetics, macro-processes occurring at the system level and microprocesses occurring at the level of subsystems are connected to each other systematically [2]. In stable systems is dominated macro-processes, which determine their evolutionary changes. If for microprocesses occurring at level of the subsystems, are peculiar even small changes in time, as a result this may lead to a revolutionary change in the system. For example, one of the major complications arising during hip arthroplasty is the wear of materials with surfaces of the pair of friction. At the certain level of head or cup wear, the whole system (endoprosthesis) loses operability. Hence, the elimination of the causes of premature failure of individual subsystems, can improve the durability of an integrated system.

Attempts to use a variety of biocompatible materials in friction pairs of total hip endoprostheses is only partially allow to solve the problem of wear, because until now are not yet developed materials, having properties similar biological and which capacity for regeneration. An alternative approach is to change the tribological properties, for example, by applying a partial regular microrelief on surfaces of friction pairs [18].

When designing implants employed within systems with variable parameters is necessary, if possible, to take into account these changes. Such approach has been used by us when creating a dynamic transpedicular system, adapted to changes of parameters of young patients in intensive growth [19].

\subsection{Synthesis of test devices}

For the most part, implants and assistive devices used for complete or partial replacement of human organs have a complicated geometrical structure and operates in conditions of complex loading. The complicated structure, the impact of complex dynamic loads, kinematic and dynamic uncertainty are typical to external limb prostheses, exoskeletons and other devices for the rehabilitation of patients with disorders of the musculoskeletal system. In this regard, the synthesis of such devices represents a nontrivial task. Search of optimal solutions of synthesis by means of analytical methods often produce on simplified models, which are not quite correctly represent the real object. To check the quality of such solutions usually is carried out an analysis of three-dimensional computer models. However, even in such cases it is very difficult to take into account all features of the designed device. The most accurate estimates can be obtained by testing prototypes on specialized testing devices. However, it requires take into account the fact that such devices must ensure complex laws of motion and loading of samples, similar to the movements of elements of the human skeleton in the process of his lifestyle. Under the project "FESTO-SYNERGY" was adopted a mechatronic 
paradigm of synthesis of test devices simulating movements of elements of the human skeleton. Such choice was based on the fact that exactly mechatronic modelling devices (simulators) are able to implement complex laws of motion of executive bodies.

In the synthesis of mechatronic modeling devices was used a system approach which allowed to formulate a conclusion that executive mechanisms within systems is acquire a new quality. This fact allows using simple mechanisms for the implementation complex laws of motion. At the same time, complex trajectories (planar or spatial) of reference points of executive bodies can be effectively implemented using mechanisms with several degrees of freedom [20]. Exactly simple mechanisms were used in the synthesis mechanical system of the mechatronic simulator for testing of friction pairs of total hip joint endoprosthesis (THJE) on wear. General view of the stand shown in Fig. 5a.

The mechanical system of the simulator is designed to implement rotational movements: FE (flexion/extension - rotation by angle $\varphi$ that provides human step during walking), AA (abduction/adduction - rotation by angle $\psi$ that provides thigh swinging in the transverse plane) and IOR (inward/outward - rotation by angle $\vartheta$ that provides thigh rotation around the vertical axis) of the tested object 1 with respect to a certain point $\mathrm{O}$, fixed relatively to the base (Fig. 5b). Additionally the mechanical system includes a device for creating the load applied to the test specimen. Thus every point on the sphere surface, center of which is at the point $\mathrm{O}$, moves along a certain trajectory (Fig. 5c) that is defined by its radius and laws of specified rotations: $F E=\varphi(t), A A=\psi(t), I O R=\vartheta(t)$ (Fig. 5d). To drive of the load device and the rotation mechanism of the carriage (EF) were selected electromechanical axes EGC-185-100-TB-KF$50 \mathrm{H}-\mathrm{GK}-\mathrm{ZUB}-2 \mathrm{MY} 2 \mathrm{X}$, and for the rotation mechanism of U-shaped bracket (AA) - the electromechanical axis EGC120-50-TB-KF-50H-GK-ZUB-2MY2X; for direct reproduction of motion IOR uses a stepper motor EMMS-ST-87-MSEB with an internal reducer [20].
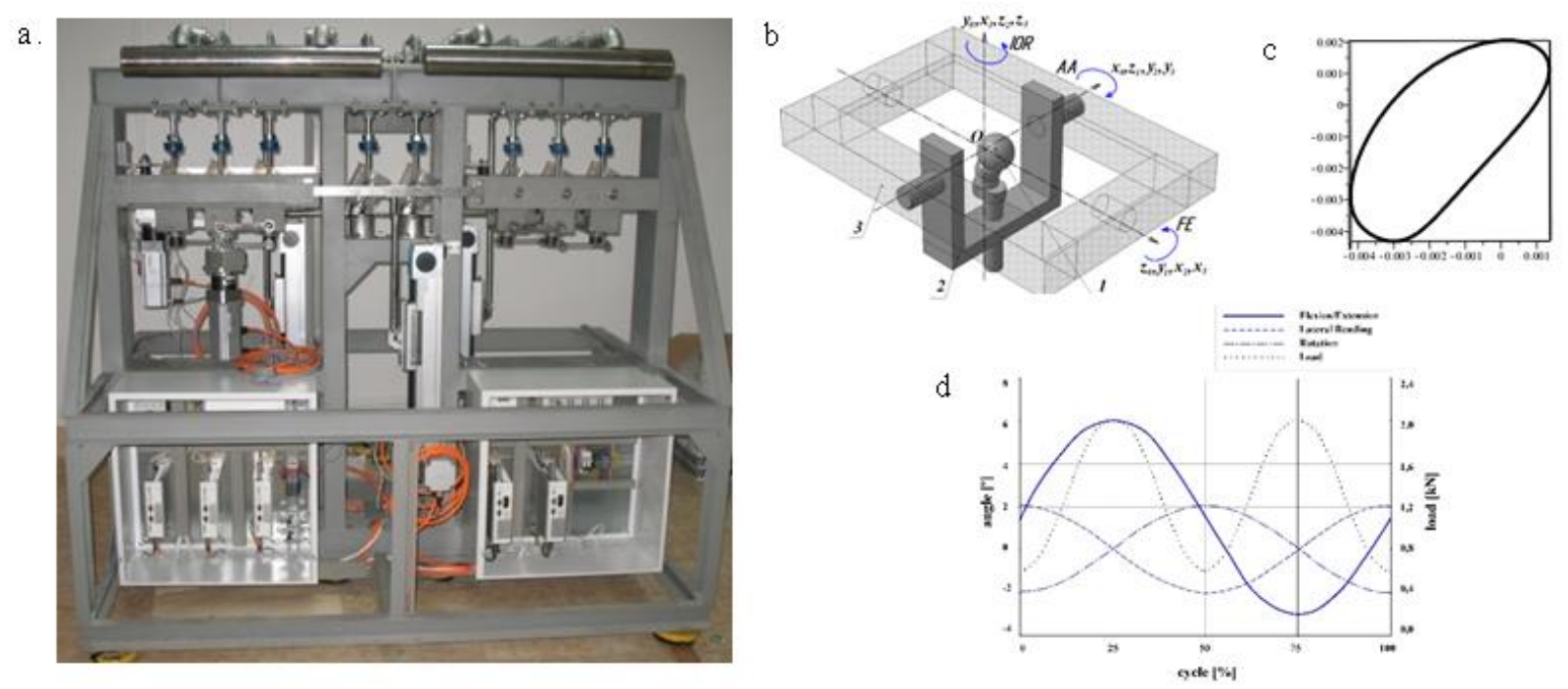

Fig. 5. (a) general view of the mechatronic simulator for testing on the wear of friction pairs hip joint endoprostheses;

(b) absolute and local coordinate systems associated with rotating links of the mechanical system: 1 - head of test specimen; 2 - U-shaped bracket; 3 - carriage; (c) trajectory of the pole on the head of tested endoprosthesis; (d) graphs of laws load changes and movements of the test specimen on three degrees of freedom

The module of the simulator motion control is built on the basis of industrial controllers of FESTO. It provide movement of tested objects on the given trajectory and supplies the required load. The module consists of terminal CPX-CEC-C1-M1, two program logic controllers (PLC) of servomotors CMMP-AS and three PLC of stepper motors CMMS-ST. The terminal is a 32-bit PLC designed to control by controllers of drives through CAN-Bus. This controller is programmed according to the standard IEC 61131-3 and supports SoftMotion PLCOpen. Its main tasks - to provide synchronous movements. Programming and program settings are carried out through the simulator control module in the environment CoDeSys. PLC of the drives serve as slave devices and perform commands of CPX terminal according to protocol CANOpen. The initial setup of controllers is carried out through the control module in the Festo Configuration Tools (FCT).

The use of presented above structure allowed to create the stand-simulator that can to implement many complex laws of motion of of test samples. Originally, the stand was designed for THJE tests on wear in accordance with the requirements of ISO/DIS 14242-1. However, it can be considered as a universal device and at the insignificant modification it can be used to testing endoprostheses of intervertebral discs [21], spherical joints of vehicle suspensions [22] and for other purposes.

Based on mechatronic paradigm have been created and are in the process of designing other devices, designated to testing various medical devices. For example, on the base of the Stewart platform with driving modules 
FESTO was created a multifunctional mechatronic stand for testing artificial feet used in transfemoral and transtibial prostheses (Fig. 6a,b). Such variant of design was chosen in view of the fact that the Stewart platform, as a mechanism of parallel structure, is a rigid mechanical system able to implement a wide range of movements of the mobile platform. This provides high precision of implemented movements [23].
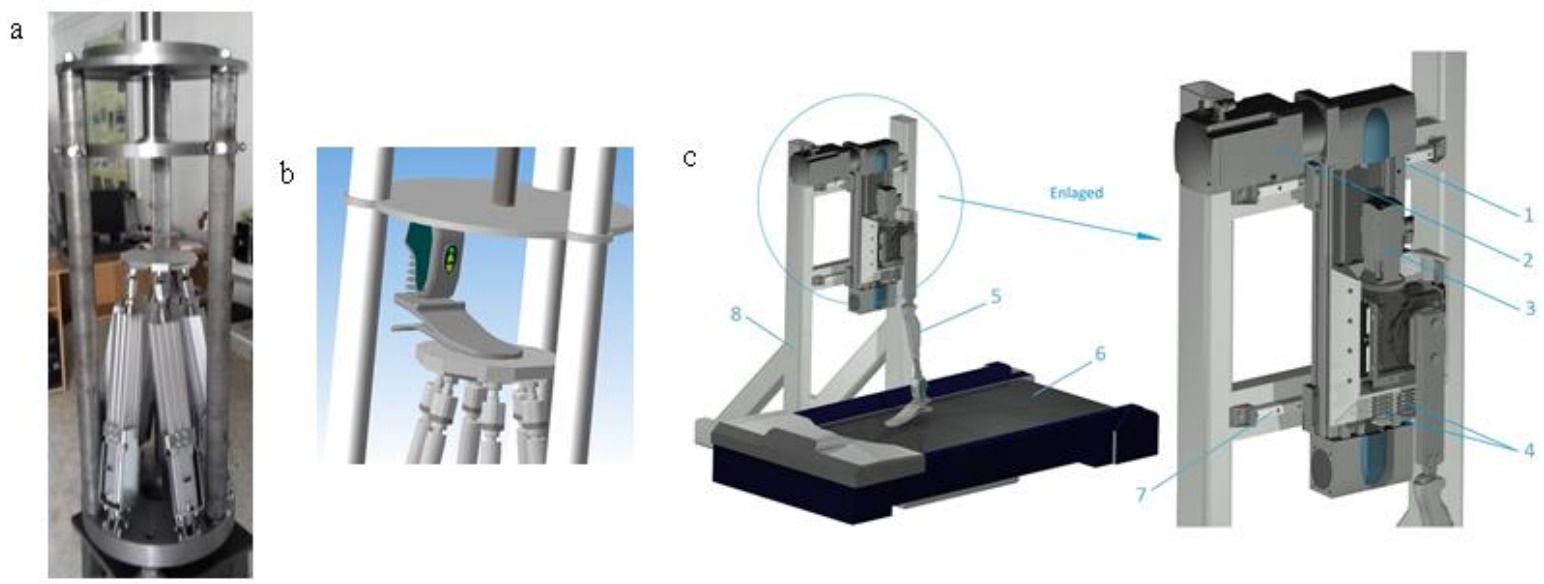

Fig. 6. (a) general view of the simulator for testing artificial feet; (b) fragment of 3D-model of the simulator at testing of artificial foot; (c) 3D model of the test bench for testing modules and control systems of transfemoral prostheses: 1 horizontal supporting beam; 2 - electromechanical axis with toothed belt; 3 - femoral component; 4 - springs; 5 prosthesis model; 6 - jogging track; 7 - rigid horizontal rails; 8 - rigid frame

On the Fig. 6c shown a 3D-model of the stand for testing modules and control systems of microprocessor controlled transfemoral prostheses. The mechanical system of the stand also represent synergistically connected modules of movements, which designed on the base of FESTO electromechanical axes. This stand allows with the high precision simulate the walking process of amputee with unilateral transfemoral amputation.

The stand working under the influence of two formally independent control systems. One of them is designed to control movements of the femoral component that simulates the movement of the stump of the amputee, and the second is used to the control of an artificial knee and artificial ankle. During the work each of these systems has certain influence on the other and conversely. Thus are realized the natural controlled motions of the prosthesis, similar to movements of amputee when walking on the prosthesis.

\section{Results and discussion}

In this work, we present some results implemented in the frame of project "FESTO-SYNERGY" and its component - engineering biomechanics. Each of presented here devices was constructed based on the systematic approach. For example, all implantable devices are considered as subsystems of the process of patients rehabilitation. They are represent subsystems of surgery processes and after implantation act as subsystems within the integrated biological system - human, actively interacted with the environment in the process of rehabilitation. Implantable devices must, if necessary, be easily removed from the human body, have the ability to adapt to changes in the body and, as foreign bodies, to have the least adverse effect on the organism.

When implants designing, the engineer, according to the accepted concept of synthesis, must always strive to create a product that is closest in character to their biological counterparts. If it is not possible to achieve such objectives, the implant has to perform its functions with the highest possible quality.

To evaluate the functional performance of new and existing medical devices and verify their compliance with existing standards are needed specialized test devices. Usually the executive bodies of such devices must perform complex movements in conditions of complex loads applied to the test specimen. We have adopted a mechatronic paradigm, in the frame of which was created a series of test devices with characteristics not inferior to the best modern counterparts. All developed by us devices can be considered as universal and after irrelevant upgrading, they can be used in testing various products and complexes in the substantially different conditions. This is due, primarily, by the ability of mechatronic systems to the quick reconfiguration.

Successful realization of a number of projects in the field of engineering biomechanics became possible due to the joint work of surgeons and engineers. The interaction process of specialists in different fields of activity was limited on the level of problems formulation (with one hand) and on the level of assessment of the feasibility of these tasks at the present level of technological development (the other side). Such process can be considered as iterative as in each of the realized projects ultimately were used the ideas adopted in result of certain compromises on the one and on the other hand. 
In perspective in the frame of the project "FESTO-SYNERGY" planned to create a low-budget transfemoral prosthesis with the microprocessor intelligent control. In tests of the main modules and control systems will be used test devices presented in this work.

\section{Acknowledgements}

The authors express their sincere gratitude to the leadership of the concern FESTO and personally to Dr. W. Stoll for the provided for this work equipment and software. Partially this work has been funded by the Ministry of Education and Science of the Russian Federation in the framework of the base part of State order in the field of scientific activity with the registration No. 115041610028.

\section{References}

[1] N.A. Bernstein, On the Construction of Movements, Medgiz, Moscow, 1947.

[2] H. Haken, Synergetics, Mir, Moscow, 1980.

[3] H. Haken, Information and Self-Organization: a Macroscopic Approach to Complex Systems, third ed., Springer, Berlin, Heidelberg, New York, 2006.

[4] M.L. Latash, J.P. Scholz, G. Schöner, Toward a New Theory of Motor Synergies, Motor Control. 11(3) (2007) 276-308.

[5] S.E Engelbrecht, Minimum Principles in Motor Control, J of Math. Psych. 45 (2001) 497-542.

[6] R. Rosen, The Optimality Principle in Biology, Mir, Moscow, 1969.

[7] N. Rashevsky, Mathematical Biophysics, Dover, New York, 1960.

[8] G. Veyl, Symmetry, Science, Moscow, 1969.

[9] I.F. Obraztsov, M.A. Hanin, Optimal biomechanical system, Medicine, Moscow, 1989.

[10] S.J. Maynard, Optimization Theory in Evolution, Annual Rev. of Eco. Sys. 9 (1978) 31-56.

[11] R. Axelrod, W.D. Hamilton, The Evolution of Cooperation, Science, 11, (1981) 1390-1396.

[12] M. Daly, M. Wilson, Sex, Evolution, and Behavior, PWS-Kent, Boston, 1983.

[13] S.V. Petukhov, Biomechanics, Bionics and Symmetry, Science, Moscow, 1981.

[14] O. Bryekhov, O. Poliakov, M. Kalinin, New Technical Aspects of Minimal Invasive Posterior Interbody Fusion, Abst. Vol. of the 1-st WCMISST, Honolulu, Hawaii, USA, 2008.

[15] A.V. Kovalenko, Evaluation Stress-Strain State of Bearing Elements Transpedicular Systems, Bul. SevNTU, 120 (2011) 181-186.

[16] A.V. Kovalenko, A.M. Poliakov, Analysis Stress-Strain State of New Monoaxial Transpedicular Screw, Bul. SevNTU, 110 (2010) 238-240.

[17] A. Poliakov, V. Pakhaliuk, M. Kalinin, M. Kolesova, V. Kramar, O. Kovalenko, System Analysis and Synthesis of Total Hip Joint Endoprosthesis, Procedia Eng., 100 (2015) 530-538.

[18] V. Pakhaliuk, A. Poliakov, M. Kalinin, V. Kramar, Improving the Finite Element Simulation of Wear of Total Hip Prosthesis' Spherical Joint with the Polymeric Component, Procedia Eng., 100 (2015) 539-548.

[19] A.M. Poliakov, Y.V. Pashkov, M.I. Kalinin, O.N. Bryekhov, O.V. Kovalenko, V.V. Volkov, Anatomically Adaptable Dynamic Transpedicular System for Functional Restoration of Spine, An. of DAAAM for 2010 \& Proc. of the 21st Int. DAAAM Symp., 21(1) (2010) 0815-0816.

[20] A. Poliakov, M. Kolesova, O. Chepenyuk, The Concept of Synthesis Hinged Mechanisms as a Part of Mechatronic Systems, J Robot Mech Eng Resr 1(2) (2015) 1-11.

[21] A. Poliakov, V. Pakhaliuk, P. Shtanko, O. Bryekhov, O. Kovalenko, I. Desyatov, A Novel Fatigue Testing Machine for Spinal Fixation Systems Under Various Conditions of Loading, SAUFBE Bull, 16(3) (2012) 157163.

[22] A. Poliakov, V. Pakhaliuk, V. Lazarev, P. Shtanko, Y. Ivanov, Stand and Control System for Wear Testing of the Spherical Joints of Vehicle Suspension at Complex Loading Conditions, Cont. and Aut. Theory for Trans. Appl., 1(1) (2013) 106-111.

[23] B. Dasgupta, T.S. Mruthyunjaya, The Stewart Platform Manipulator: a Review, Mech. and Machine Theory, 35(1) (2000) 15-40. 